livraisons

d'Histoire

de l'Architecture

\section{Livraisons de l'histoire de l'architecture}

32 | 2016

Les représentations de l'architecture

\title{
Antonio Sant'Elia et La Città Nuova : représenter la ville moderne
}

Antonio Sant'Elia and La Città Nuova: representing the modern city

Sant'Elia und La CittàNuova. Darstellungen der modernen Stadt

\section{Viviana Birolli}

\section{(2) OpenEdition}

Journals

Édition électronique

URL : http://journals.openedition.org//ha/641

DOI : 10.4000/lha.641

ISSN : 1960-5994

Éditeur

Association Livraisons d'histoire de l'architecture - LHA

Édition imprimée

Date de publication : 31 décembre 2016

Pagination : 89-104

ISSN : 1627-4970

Référence électronique

Viviana Birolli, "Antonio Sant'Elia et La Città Nuova : représenter la ville moderne », Livraisons de I'histoire de l'architecture [En ligne], 32 | 2016, mis en ligne le 31 décembre 2018, consulté le 10

décembre 2020. URL : http://journals.openedition.org//ha/641 ; DOI : https://doi.org/10.4000//ha.641 
Par Viviana BIROLLI

\section{ANTONIO SANT'ELIA ET LA CITTÀ NUOVA : REPRÉSENTER LA VILLE MODERNE}

Antonio Sant'Elia (1888-1916), "architecte futuriste", meurt au front le 10 octobre 1916 à 28 ans, touché par une balle pendant la huitième bataille de l'Isonzo, alors qu'il était enrôlé dans le bataillon Volontaires Cyclistes Automobilistes avec, entre autres, Filippo Tommaso Marinetti, Umberto Boccioni et Luigi Russolo ${ }^{1}$. L'histoire se souviendra de lui avant tout pour le manifeste L'Architecture futuriste, lancé à Milan le 11 juillet 1914 par la Direction du Mouvement futuriste.

Depuis, ce tract de quatre pages illustré par six dessins de La Città Nuova est rentré à plein titre dans une histoire utopique des métropoles du futur : tout au long du $\mathrm{XX}^{\mathrm{e}}$ siècle, la verticalité exaspérée de cette métropole gigantesque se déployant exclusivement sur le papier a inspiré l'imaginaire aussi bien d'architectes que de cinéastes ${ }^{2}$.

Cependant, le corpus d'œuvres confié à l'histoire par Sant'Elia excède la série de dessins iconiques de La Città Nuova : s'il n'a pratiquement rien bâti, cet " architecte sans architectures " est l'auteur de plus de 300 dessins $^{3}$ - détails architecturaux, projets, esquisses, parmi lesquelles la série des Dynamismes architectoniques (191314) - et d'un texte intitulé Messaggio, publié en mai 1914 à l'occasion de la première exposition du groupe Nuove Tendenze à la Famiglia Artistica de Milan (20 mai-10 juin 1914).

Une reconsidération de l'ensemble de la production de Sant'Elia, à l'aune de son trajet créatif mais aussi du contexte culturel et architectural dans lequel il opérait, peut permettre de redonner une unité à son parcours et une profondeur historique au geste utopique de son manifeste. Ce retour sur les documents sera aussi le point de départ pour conduire une analyse de la valeur rhétorique des choix graphiques d'une série de dessins conçus pour développer sur un plan éminemment représentatif et idéologique une image totale de la ville nouvelle de la modernité. De cette Città Nuova, l'utopie urbaine de Sant'Elia s'efforce en effet d'exprimer aussi bien l'aspect visuel que les logiques de fonctionnement, décrivant et prescri-

1. Bertoni Alberto, Filippo Tommaso Marinetti. Taccuini 1915-1921, Bologne, Il Mulino, 1987.

2. De Michelis Marco, La Città Nuova oltre Sant'Elia, 1913-2013. Cento anni di visioni urbane, catalogue de l'exposition, Villa Olmo, Côme, 24 mars-14 juillet 2013, Milan, Silvana, 2013.

3. Caramel Luciano, Longatti Alberto, Antonio Sant'Elia. L'opera completa, Milan, Arnoldo Mondadori, 1987. 
vant sur le papier non seulement le style de vie de l'homme nouveau, mais aussi le type de regard que ce dernier devait porter sur la métropole démesurée qui était à la fois sa retombée naturelle et son berceau artificiel.

\section{Une réception critique ambivalente}

De jeune architecte "romantique " empreint de modèles traditionnels à prophète indépassé de la modernité urbaine, la figure de Sant'Elia a fait l'objet de lectures ambivalentes au cours du $\mathrm{XX}^{\mathrm{e}}$ siècle.

Aux années 1930, c'est Marinetti qui soumet pour la première fois l'architecte de Côme à une récupération de type "agiographique » censée en faire le prophète italien du rationalisme international : la fondation d'une revue dédiée ${ }^{4}$ à son nom, la mise en scène de spectacles ${ }^{5}$ et la réalisation, de la part de Giuseppe Terragni, d'un monument aux morts de la Première Guerre mondiale de Côme inspiré d'une esquisse de Sant'Elia pour une tour-phare (1914), font partie de la récupération rétrospective de la valeur prophétique de l'œuvre de l'architecte orchestrée par l'impresario du futurisme.

Vingt ans plus tard, en 1956, l'architecte Bernasconi redécouvre ${ }^{6}$ le texte du Messaggio rédigé par Sant'Elia deux mois avant le lancement de son manifeste et son adhésion au futurisme: les analogies et les différences entre ces deux textes se posent alors à l'origine d'une polémique sur le caractère autographe du manifeste. Ce texte s'avère en effet être le fruit d'une série d'interventions opérées par Buggelli, Nebbia et Marinetti, dans le but de souligner les éléments de nouveauté et l'effet de choc des intuitions sant'eliennes : parmi ces ajouts figurent notamment quelques-uns des points les plus novateurs du manifeste, entre autres l'affirmation "Les maisons dureront moins que nous. Chaque génération devra fabriquer sa propre ville", qui anticipe les projets d'architecture évolutive de groupes tels qu'Archigram. D'autre part, ce type d'opérations de relookage rhétorique était à l'ordre du jour chez les futuristes ${ }^{7}$, au nom d'un " art de faire les manifestes " dont Marinetti seul détenait l'apanage. À partir de ces discordances textuelles, la critique italienne des années 1950 aboutit à mettre en doute l'adhésion de Sant'Elia au futurisme $^{8}$, mais aussi la valeur de nouveauté de ses dessins, lus comme des rêveries de jeunesse destinées à ne rien apporter à une histoire de l'architecture bâtie.

Cependant, le mouvement Nuove Tendenze auquel Sant'Elia adhérait à l'époque de la publication du Messaggio se revendiquait ouvertement comme l'«aile droite

4. Sant'Elia, revue dirigée par Mario Somenzi et Angiolo Mazzoni à partir d'octobre 1933.

5. Entre autres Ricostruire l'Italia con architetture futuriste Sant'Elia, "divertissement représentable en de nombreux synthèses ", rédigé par Marinetti en 1930.

6. Bernasconi Giovanni "Il messaggio di Antonio Sant'Elia del 20 maggio 1914 ", Rivista Tecnica della Svizzera Italiana, juillet 1956, p. 145-152.

7. Pratella F. Balilla, Autobiografia, Milan, Pan, 1971, p. 101-103.

8. Zevi Bruno, "Sant'Elia non era futurista ", L'espresso, II, n. 36, Rome, septembre 1956 ; "I disegni smentiscono il manifesto futurista ", L'Espresso, II, n. 44, Rome, octobre 1956. 
et modérée » du futurisme. Soulignant l'impact profond du message futuriste sur tout le climat culturel italien du début du $\mathrm{XX}^{\mathrm{e}}$ siècle, Reyner Banham ${ }^{9}$ réduit dès lors cette polémique sur la paternité du texte à une "affaire toute italienne », consignant l'œuvre de l'architecte à une histoire internationale des visions de la ville du futur, destinée à influencer de manière durable les imageries de la modernité urbaine. C'est au titre de cette histoire imagée que, depuis les années 1960, l'œuvre de Sant'Elia ne cesse de faire l'objet de récupérations critiques et visuelles.

Une réconsideration du caractère ambivalent des lectures de La Città Nuova à l'aune du parcours de formation de l'architecte de Côme permet d'appréhender Sant'Elia comme l'un des interprètes de la modernité architecturale de l'Italie du Nord du début du $\mathrm{XX}^{\mathrm{e}}$ siècle en tant que moment de "crise ": métamorphose abrupte des modèles urbains intervenant dans un contexte encore lié à des modèles traditionnels, où l'accès au panorama international était aussi difficile que limité. "Nous sommes en retard", déclarait Colajanni à propos de l'Italie au tournant du $\mathrm{XX}^{\mathrm{e}}$ siècle $^{10}$ : c'est précisément à la lumière de ce contexte que, bien au délà de leur caractère provocateur et promotionnel, les manifestes futuristes prennent toute leur valeur de laboratoires de l'homme nouveau, conçus pour faire exister la modernité dans le geste même de leur énonciation.

\section{Milan, 1907-1913 : le parcours de formation de Sant'Elia}

Lorsque, âgé à peine de 20 ans, Antonio Sant'Elia débarque à Milan, la « capitale morale " de la naissante Italie industrielle est une ville en plein essor : la fondation des premiers grands établissements d'industrie automobile et métallurgique accélère la croissance démographique et le développement des banlieues ouvrières. La construction de centrales électriques et de quartiers populaires est une question à l'ordre du jour : ainsi, dès son arrivée à Milan Sant'Elia collabore à l'élaboration du nouveau plan urbain de la ville, qui sera présenté par les ingénieurs Masera et Pavia en 1909. En 1902, la Première Exposition internationale d'art décoratif moderne de Turin contribue à diffuser en Italie les influences de l'art nouveau, tandis que l'Exposition internationale de Milan de 1906 met les infrastructures de transport au centre des problématiques architecturales de la modernité.

Tandis que ces vents de renouveau traversent l'Italie du Nord, l'Académie de Brera où Sant'Elia se forme et les architectes en vogue - dont Coppedé, Sacconi et Calderini - continuent toutefois de prôner un éclectisme hybride basé sur la variation fantastique et formaliste de styles décoratifs anciens et exotiques appliqués à des structures traditionnelles. Véritable demeure néo-médiévale au cœur technologique, la centrale hydro-électrique Taccani de Gaetano Moretti (1903-1906,

9. Banham Reyner, "Poetica di Sant'Elia e ideologia futurista ", The architectural Review, Londres, mai 1955 ; Theory and Design in the First Machine Age, Londres, Architectural Press, 1960.

10. Colajanni Napoleone, L'Italia nel 1898 (tumulti e reazione), Milan, Società editrice lombarda, 1989, p. 28. 
Trezzo d'Adda) illustre bien le retard substantiel des modèles visuels de l'architecture italienne.

Le parcours de recherche de Sant'Elia entre 1907 et 1913 reflète l'ensemble de tensions qui parcouraient le contexte architectural de l'Italie du Nord de l'époque, entre permanence de modèles anciens et élans vers le futur. Ainsi, ses deux premiers projets publiés - le projet d'un pavillon moderne présenté en 1908 à un concours de la coopérative Milanino et les croquis pour une maison parus en janvier 1909 dans la revue $L a$ Casa - attestent d'une épuration visuelle progressive par rapport aux premières copies de détails décoratifs de 1907, mais aussi d'une inspiration visuelle directe aux modèles de la Wagnerschule et à l'œuvre d'Olbrich, que Sant'Elia connaissait grâce au volume Die Wagnerschule 1902 (Vienne, 1903).

Entre 1909 et 1910, l'architecte réalise de nombreux croquis pour des édifices monumentaux qui préfigurent déjà quelques-uns des éléments formels de La Città Nuova (ill. 1) : du contrefort à la tour, en passant par la pyramide. Toutefois, ces premières épreuves n'échappent pas à la permanence de motifs décoratifs liberty, orientaux, exotiques et médiévaux prolongeant la veine éclectique de l'architecture italienne de l'époque, comme en atteste entre autres son projet pour le cimetière de Monza (1911-1912) (ill. 2). La recherche d'un effet théâtral, obtenu par l'adoption d'un point de vue en contre-plongée et en biais, est un autre aspect marquant de ces esquisses : dépourvus d'une destination fonctionnelle claire, ces édifices se dressant au sommet d'imposants escaliers et rassemblant de près à des édifices de culte - à mi-chemin entre un temple, une église et une mosquée - pourraient dès lors suggérer l'idée d'une origine monumentale et scénographique de la syntaxe formelle de La Città Nuova.

L'oscillation entre intuitions modernes et permanence de modèles traditionnels est particulièrement évidente dans l'écart visuel qui sépare les recherches personnelles et les travaux professionnels de l'architecte : ainsi, la Villa Elisi commanditée par Romeo Longatti en 1912 - le seul projet bâti de l'architecte de Côme - se caractérise par une structure on ne peut plus classique, si ce n'est pour une frise décorative inspirée de l'œuvre de Klimt, tandis que le projet d'Arrigo Cantoni pour la Cassa di Risparmio de Vérone, auquel Sant'Elia collabore entre 1913 et 1914, confirme la continuité de la veine éclectique et décorative dans l'architecture italienne de l'époque.

Si l'évacuation progressive des motifs décoratifs atteste de la maturation d'un langage moderne chez l'architecte de Côme déjà à partir de 1912, c'est en 1913 que ses recherches arrivent à un tournant: Sant'Elia développe alors une série de dessins qui anticipent de manière directe les intuitions de La Città Nuova. Baptisés dans les années 1930 par le futuriste Escodamé Dynamismes architectoniques en hommage à l'œuvre de Boccioni, ces dessins de purs solides géométriques aux surfaces nues traduisent sur le papier l'élaboration d'un véritable vocabulaire formel de la ville moderne. Comme le souligne bien Portoghesi ${ }^{11}$, c'est une métaphore

11. Portoghesi Paolo, "Il linguaggio di Sant'Elia ", Controspazio, IV, n. 4-5, Milan, avril-mai 1971, p. 30 . 


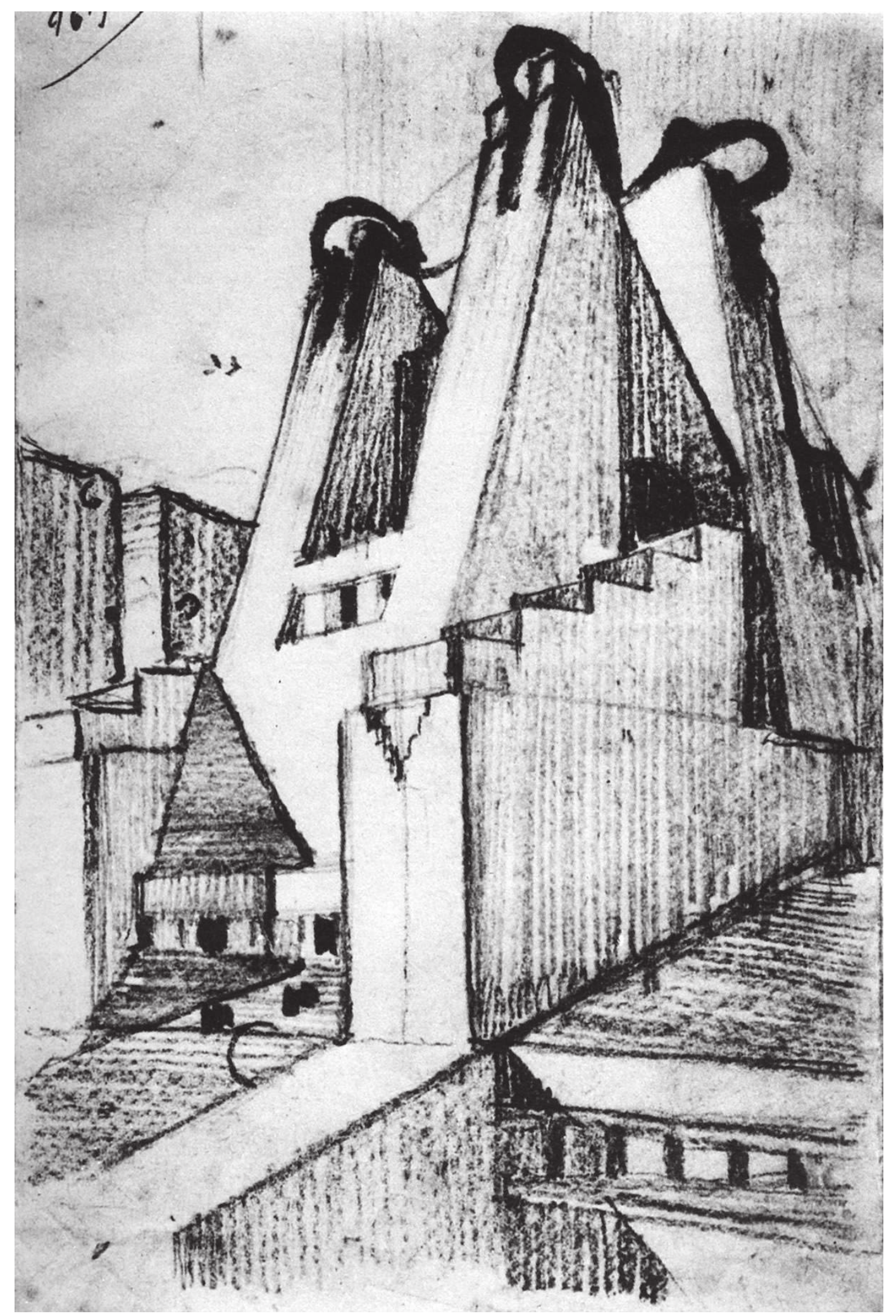

Ill. 1 : Antonio Sant'Elia, «Edificio monumentale », 1909, crayon sur papier, 17,7 × 11,9 cm, Museo civico, Crémone. 


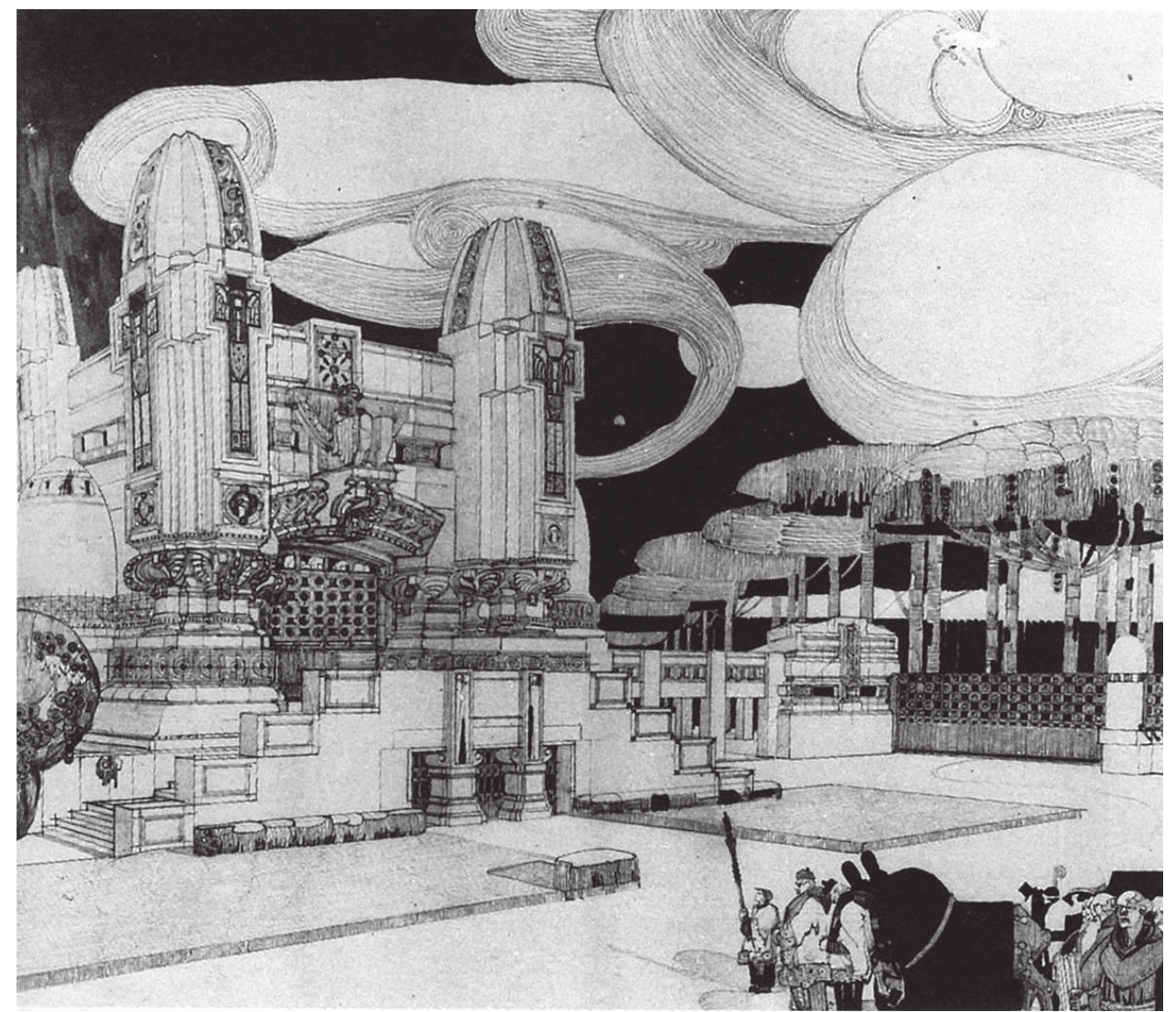

Ill. 2 : Projet pour le nouveau cimetière de Monza, Antonio Sant'Elia, 1911-1912, La Lombardia, 17 octobre 1912.

linguistique qui sous-tend cette phase de transition de la réflexion de Sant'Elia : les Éléments architectoniques des années 1913 et 1914 peuvent dès lors être lus comme étant le creuset de la construction d'un "schème discursif " autonome de la ville moderne, articule au travers d'une série d'éléments formels récurrents - la pyramide, la tour, le L renversé, le contrefort - et d'un ensemble de règles de composition - symétrie, répétition modulaire, obliquité, superposition (ill. 3). Ces essais de purisme géométrique radical incarnent tout l'effort de libération de Sant'Elia de l'éclectisme décoratif et des structures traditionnelles de l'architecture italienne de l'époque; mais ils témoignent aussi de la racine moins fonctionnelle que formelle, moins technique que graphique, de sa conception de la ville nouvelle.

C'est donc par abstraction et stylisation des profils linéaires des édifices monumentaux des années précédentes que Sant'Elia aborde le thème de la ville moderne : sans portes ni fenêtres, dépourvus d'une destination fonctionnelle précise, ces squelettes de bâtiments solitaires sont autant d'espaces conceptuels disponibles, dans lesquels émerge progressivement une réflexion sur les matériaux et les besoins de la métropole industrielle. Au fil de projets de plus en plus complexes, Sant'Elia brosse ainsi sur ces feuilles une étude expérimentale des usages du fer, du verre et du ciment à destination des bâtiments pour le plus grand nombre - théâtres, usines, gares. Les esquisses qu'il consacre en 1913 à une centrale électrique confirmeraient 


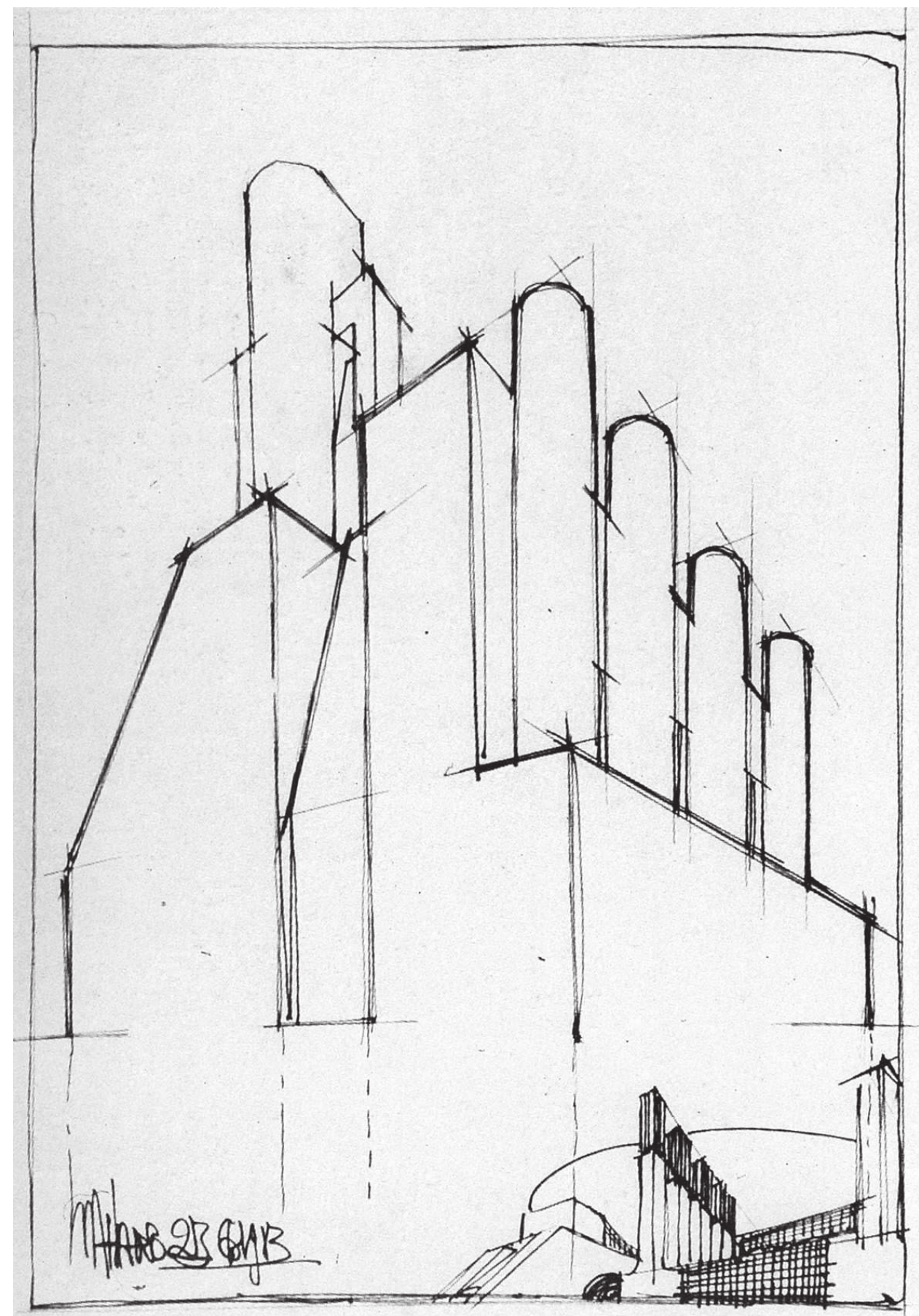

Ill. 3 : Antonio Sant'Elia, "Studi per edifici », 1913, encre noire sur papier, 27,7 × 19,5 cm, Museo civico, Côme.

néanmoins l'idée d'une racine prioritairement graphique de sa réflexion sur la ville moderne (ill. 4) : les équipements techniques y sont imprécis et approximatifs, laissant aux effets visuels le soin de concrétiser en même temps la fonction et l'impact expressif et suggestif de l'édifice. Représentée en biais, à partir d'un point de vue en contre-plongée dramatisant les lignes de fuite perspectives et le dynamisme des fils aériens, cette centrale démesurée se donne dès lors à lire sur le papier comme 


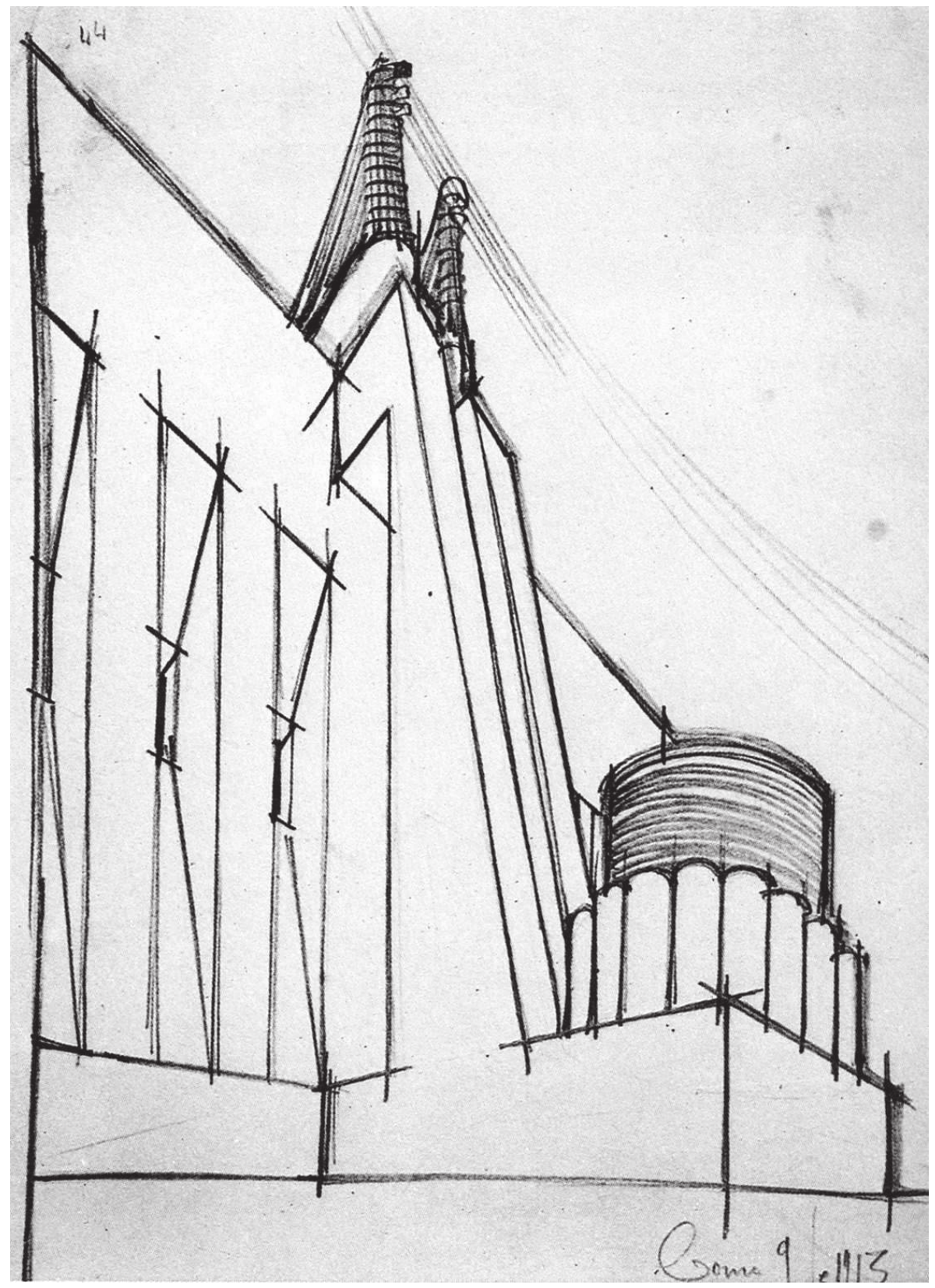

Ill. 4 : Antonio Sant'Elia, "Studio per una centrale elettrica ", [1913], encre noire et orange sur papier, $29,5 \times 19,4 \mathrm{~cm}$, Museo civico, Côme.

un véritable monument de la modernité, consacré au culte de cette "fée électricité " que, dans les mêmes années, les futuristes étaient en train d'élire en idole et moteur du monde futur.

\section{4: La Città Nuova. Représenter la ville moderne}

Quand, en 1914, Sant'Elia lance son manifeste, il postule à la racine de sa vision de La Città Nuova la rupture de toute loi de continuité historique entre l'architecture futuriste et la tradition précédente : 
«Le problème de l'architecture futuriste n'est pas un problème de remaniement linéaire. [...] Il ne s'agit pas de trouver de nouveaux profils [...] ni de déterminer des différences de formes entre l'édifice nouveau et l'édifice ancien; mais il s'agit de forger de toutes pièces la maison futuriste, de la bâtir avec toutes les ressources de la science et de la technique, en satisfaisant du mieux possible les exigences propres à nos coutumes et à notre esprit, [...] en déterminant des formes nouvelles [...], une architecture qui ait son unique raison d'être dans les conditions spéciales de la vie moderne, et sa correspondance dans une valeur esthétique propre à notre sensibilité ${ }^{12}$ ".

Les dessins qui accompagnent ce manifeste soutiennent sur le plan visuel ce mythe d'une genèse ex nibilo: les complexes productifs et les infrastructures de transport imposantes qui destinent la Città Nuova à un futur de dynamisme inébranlable sont conçus pour effacer toute dette de l'architecte de Côme vis-à-vis de la réflexion de ses homologues. Les seuls éléments de continuité qui attestent de l'unité profonde du parcours de Sant'Elia résident dans les options graphiques et représentatives de ces nouvelles formes de la modernité : de l'adoption d'un point de vue en biais, en plongée ou en contreplongée, à l'usage de coupes de cadre dramatisant la verticalité et le gigantisme des bâtiments, ces choix formels font écho aux recherches menées par l'architecte entre 1910 et 1913, dont ils prolongent l'approche scénographique et monumentale (ill. 5). En même temps, ces dessins font preuve d'un soin du détail technique inédit dans le parcours de Sant'Elia : c'est précisément cette dialectique entre valeurs expressifs et techniques qui se pose à la racine de l'ambigüité profonde et du réalisme hautement suggestif de ces projets. C'est à partir de ces indications formelles qu'il est possible de lire, en filigrane, l'ensemble de tensions qui parcourent les projets de la Città Nuova, en en faisant un point d'observation privilégié pour appréhender la transition de l'architecture italienne du début du siècle vers les nouvelles problématiques de la modernité industrielle et urbaine.

Loin d'être le fruit d'une genèse ex nibilo, La Città Nuova, et plus en général les imageries urbaines du futurisme, puisent en effet leurs racines dans un complexe de sources touchant aussi bien à l'architecture qu'à la littérature de l'époque. Ainsi, les thèmes de la métropole et de l'homme futurs occupent une large place dans les pages des écrivains italiens et étrangers du début du XX $\mathrm{XX}^{\mathrm{e}}$ siècle - de Jules Vernes à Ricciotto Canudo, en passant par Enrico Corradini et Ettore Cozzani. Plus particulièrement, les images frôlant la science-fiction de la ville futuriste comme machine gigantesque, réseau de "routes mécaniques, canalisations d'eau et d'électricité », de " vrombissements, souffles, rugissements d'hélices, fils électriques, réclames publicitaires " trouvent des antécédents précis dans The time machine (1895) de Herbert George Wells (dont la première traduction italienne parait chez Vallardi en 1900), mais aussi dans La Nuova Arma (La macchina) de Mario Morasso, publiée en 1905 dans la revue de Marinetti Poesia.

12. Sant'Elia Antonio, Manifeste de l'architecture futuriste, Milan, 11 juillet 1914, dans Lista Giovanni, Futurisme. Manifestes, documents, proclamations, Lausanne, L'Âge d'Homme, 1973, p. 233. 


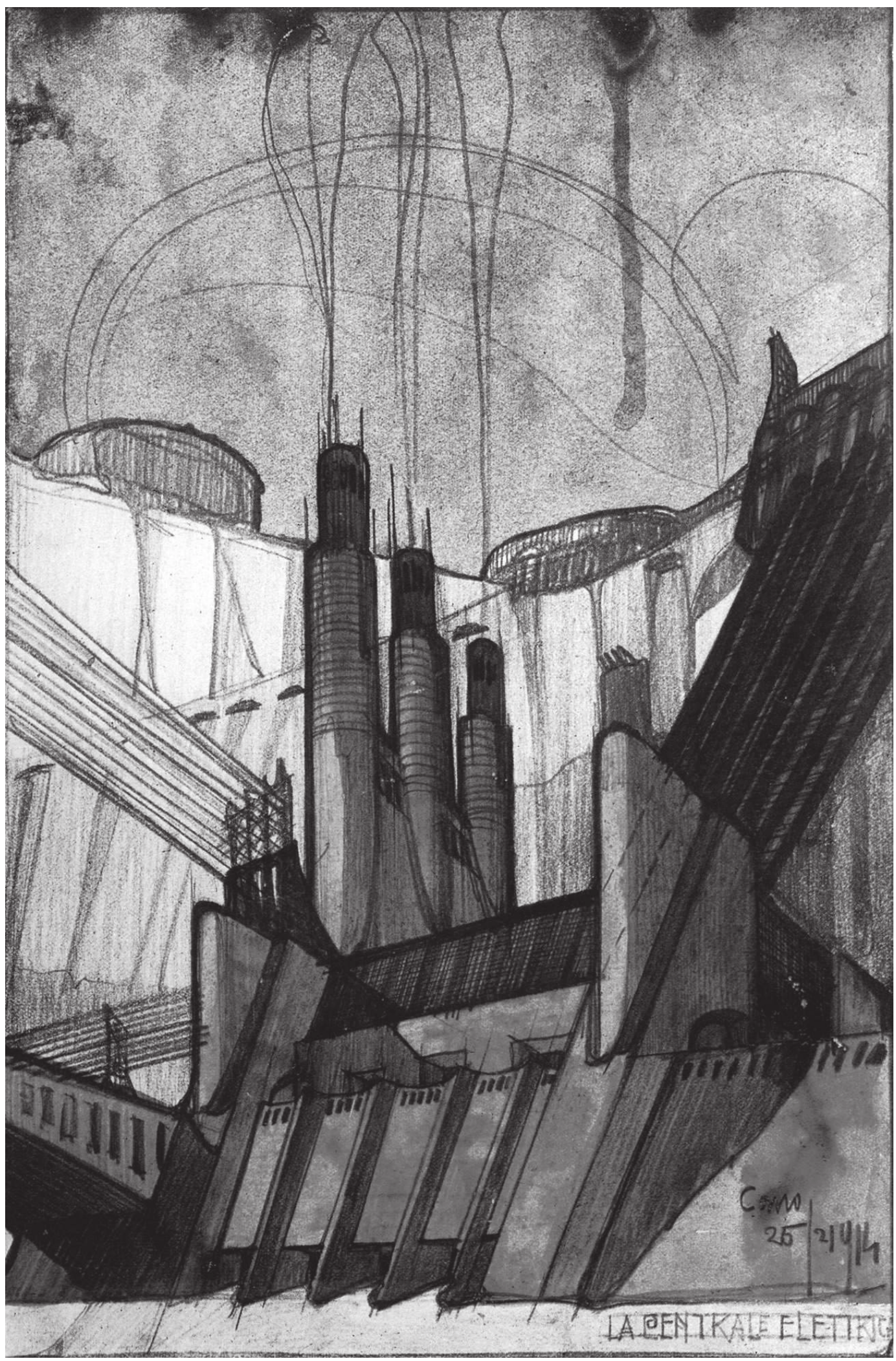

Ill. 5 : Antonio Sant'Elia, "Centrale elettrica », [1914], encre noire et gouache sur papier. 


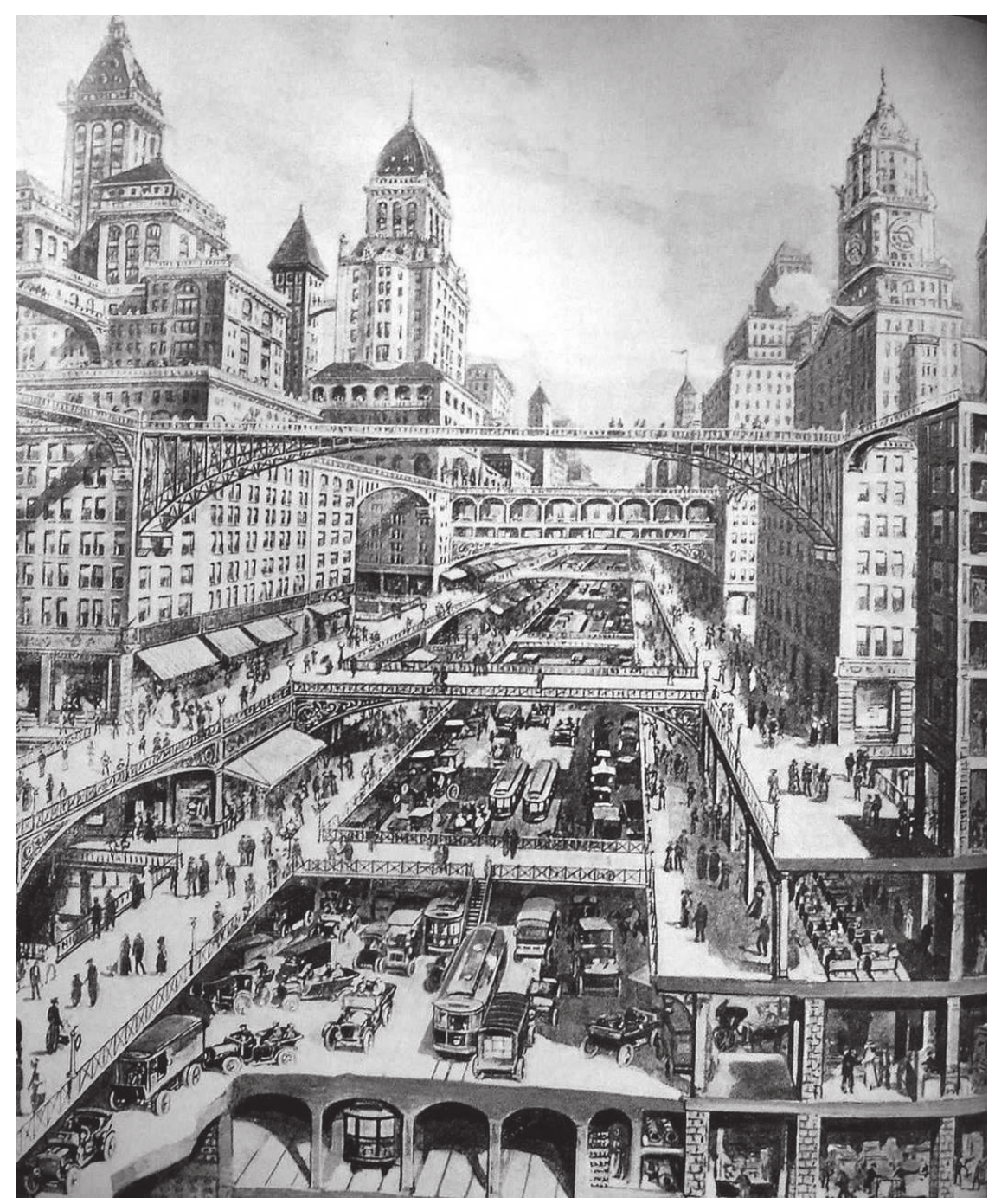

Ill. 6: Harvey Wiley Corbett, "La circolazione futura e i grattanuvole a Nova York», L'Illustrazione italiana, 31 août 1913.

Dans la même période, les premiers magasins illustrés grand public comme L'Illustrazione italiana véhiculent la pénétration de suggestions urbaines américaines - dont le gratte-nuage et l'idée d'un développement sur plusieurs niveaux du réseau de communication - par des illustrations dystopiques du New York futur signées par, entre autres, Richard Rummell et Harvey Wiley Corbett (ill. 6). Le projet (1914) de Sant'Elia pour une gare d'avions et trains sur trois niveaux (ill. 7), où l'architecte abandonne la perspective en biais qui lui était chère à la faveur d'un regard surplombant et aérien dramatisant davantage l'ampleur de la structure que sa verticalité, sont là pour montrer à quel point il est tributaire des images de Corbett.

Par ailleurs, les idéaux que depuis 1909 les futuristes opposaient au « rêve fatigué " de l'Italie vu comme "terre des morts " et " musée en plein air " ${ }^{13}$ exercent

13. "Pour les autres peuples l'Italie est encore une terre des morts, une immense Pompei blanchissant de cercueils. L'Italie tout au contraire renaît, et à son Risorgimento politique suit la renaissance 


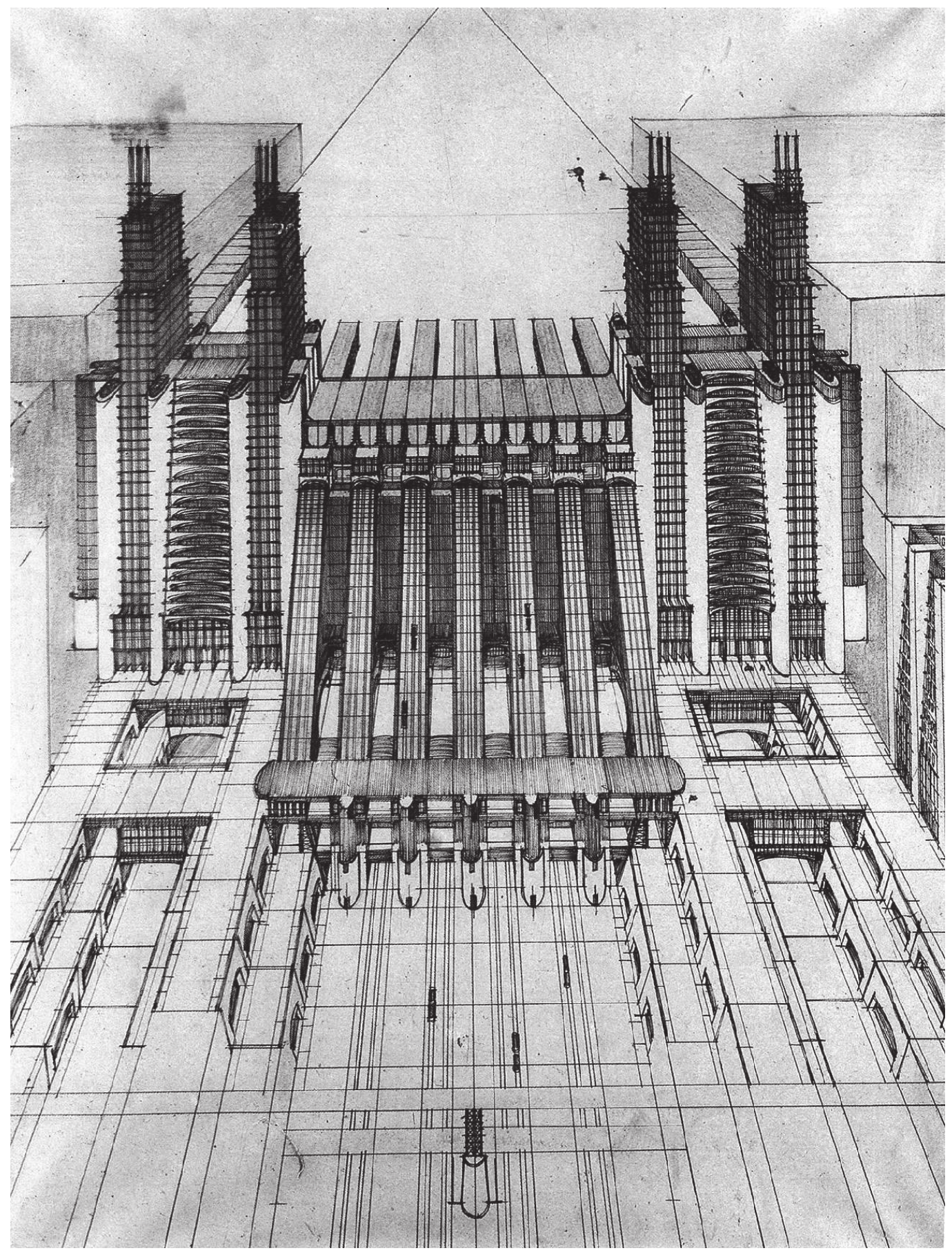

Ill. 7 : Antonio Sant'Elia, «Stazione di aeroplani e treni ferroviari con funicolari e ascensori su tre livelli stradali », [1914], encre noire sur papier.

leur influence sur l'esprit de Sant'Elia bien avant l'adhésion de l'architecte au mouvement, comme en témoignent non seulement les Dynamismes Architectoniques, mais aussi les analogies entre son Messaggio et le manifeste de l'architecture futuriste

intellectuelle "; Boccioni Umberto, Manifesto dei pittori futuristi, 11 février 1910, dans Birolli Viviana, Manifesti del futurismo, Milan, Abscondita, 2009, p. 27-33. 
rédigé par Umberto Boccioni ${ }^{14}$ entre 1913 et 1914 . Resté inédit au stade de manuscrit jusqu'aux années 1970, ce manifeste fait écho à maints égards à la recherche de Sant'Elia : du refus des styles anciens à la priorité de la fonction sur la forme, en passant par la métaphore de la machine. "Les espaces d'un édifice doivent offrir, à l'image d'un moteur, un rendement maximal ", selon l'équation "économie + utilité + vitesse ", écrit l'artiste ; "Nous devons inventer et refabriquer la ville futuriste à l'image d'un immense chantier tumultueux [...] et la maison futuriste comme une machine gigantesque », proclame à son tour l'architecte.

"Aujourd'hui nous commençons à avoir autour de nous un environnement architectural qui se développe dans tous les sens: des souterrains lumineux des grands magasins aux montées gigantesques des gratte-nuages américains, en passant par les différents niveaux de tunnels des métros " ${ }^{15}$ : prolongeant la volonté $\mathrm{du}$ peintre de La ville se lève (1910-1911) de placer le spectateur au centre du tableau, cette notion d'environnement architectural tisse un lien entre les arts plastiques et l'architecture, permettant de souligner l'unité profonde qui inspire la reconstruction futuriste de l'univers ${ }^{16}$ prônée par Marinetti. À la racine du projet futuriste dans tous ses domaines d'expression se trouve en effet le constat d'une révolution anthropologique radicale, "déterminée par tout ce qui n'existait pas auparavant " ${ }^{17}$ : si « Nous sentons que nous ne sommes plus les hommes des cathédrales, des palais, des tribunes; mais ceux des grands hôtels, des gares, des routes immenses [...] " ${ }^{18}$, c'est que, comme l'écrit Boccioni, les futuristes sont «les primitifs d'une nouvelle sensibilité centuplée ${ }^{19}$.

Ayant été conçus pour l'exposition milanaise du groupe Nuove Tendenze en dehors de toute contrainte de construction, les dessins de La Città Nuova peuvent alors mettre toutes les ressources rhétoriques de la représentation au service d'une vision totale non seulement de la ville, mais aussi de l'homme censé l'habiter et du regard que ce dernier devra porter sur une métropole qui se veut simultanément machine austère et monument suggestif de la modernité. Puisque le mode d'existence de cet homme voué au progrès est le dynamisme, sa ville ne pourra qu'être une gigantesque infrastructure de transports définie par des ponts, des ascenseurs,

14. Boccioni Umberto, Architettura futurista. Manifesto, dans Birolli Zeno, Boccioni. Gli scritti editi e inediti, Milan, Feltrinelli, 1971, v. 2, p. 36-40.

15. Ibid.

16. Du titre du manifeste lancé par Balla et Depero en 1915 ; Lista Giovanni, Futurisme, op. cit., 1973, p. 202-204.

17. Ardengo Soffici souligne bien ce lien lorsqu'il écrit : "C'est une pensée commune de dire, par exemple, que l'invention de la lampe à arche a transfiguré les vies de nos villes [...]. Ce qui ne se souligne jamais assez est à quel point, sous l'impression d'autant de changements et de spectacles nouveaux qu'en dérivent, notre raison ait été modifiée - et, avec elle, toute notre vision de l'univers "; Soffici Ardengo, Primi principi di un'estetica futurista, 1920, dans Drudi Gambillo Maria, Fiori Teresa, Archivi del futurismo, Rome, De Luca, 1958, v. I, p. 582.

18. Sant'Elia Antonio, Manifeste de l'architecture futuriste, Milan, 1914, dans Lista Giovanni, op. cit., 1973, p. 233.

19. Boccioni Umberto, Manifeste des peintres futuristes, juillet 1910, dans Lista Giovanni, Futurisme, op. cit., 1973, p. 165. 


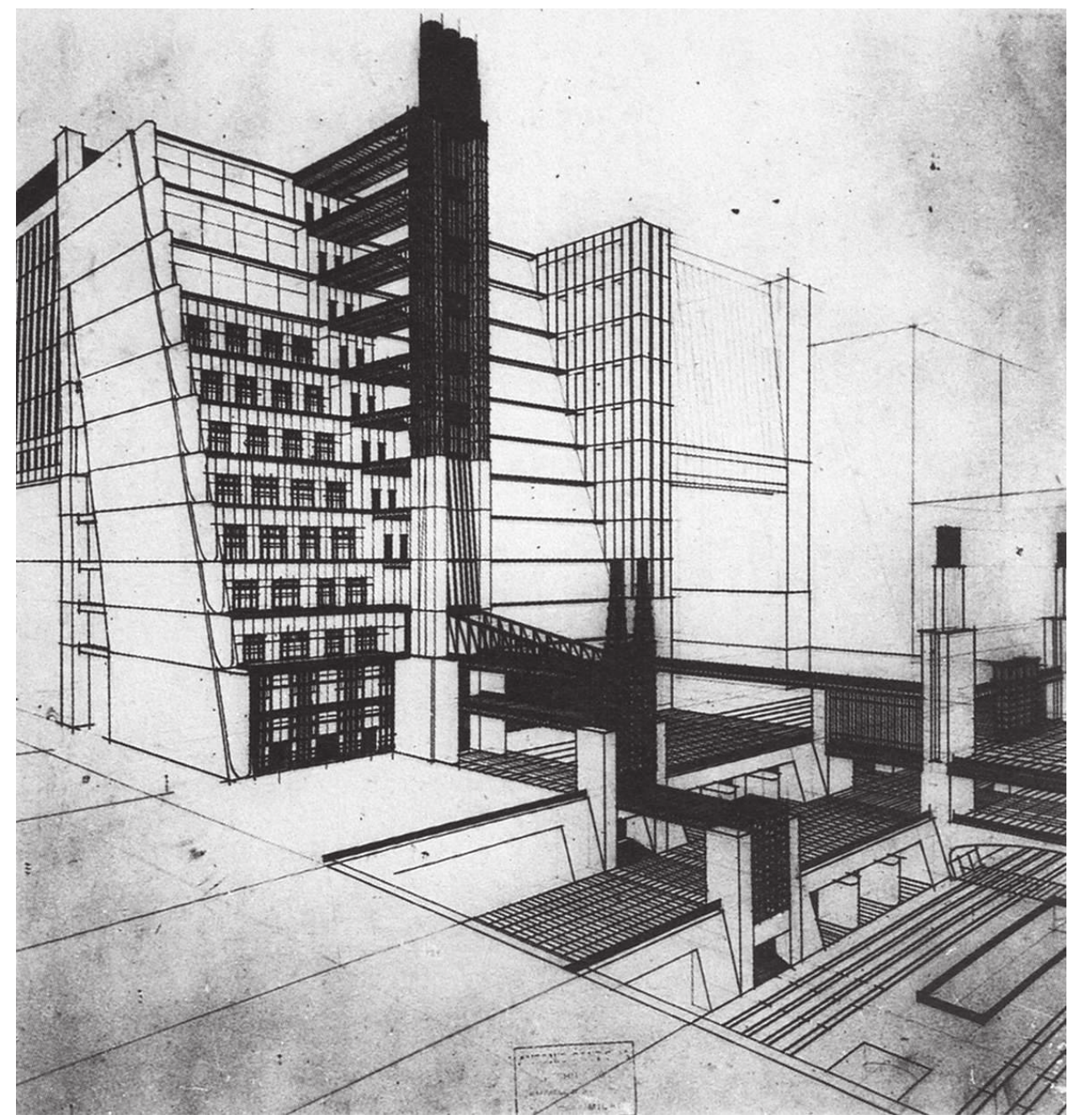

Ill. 8 : Antonio Sant'Elia, "La Città Nuova : casa a gradinata con ascensori dai quattro piani stradali ", 1914, crayon noir et encre noire sur papier, $56 \times 55 \mathrm{~cm}$, Museo civico, Côme.

des gares et des voies routières auxquelles les perspectives en biais et l'usage expressif des lignes et de la couleur assurent une valeur symbolique inédite (ill. 8).

De ce point de vue, La Città Nuova de Sant'Elia est moins un projet architectural que le théâtre d'une nouvelle idéologie urbaine, faisant de la ville une articulation complètement extériorisée de trajectoires pour un homme défini par son propre mouvement : il n'y a naturellement pas de jardins ni de places dans cette ville où tout est dynamisme sans répit. Il n'y a pas non plus de place pour l'homme dans cette métropole vide et silencieuse, écrin étincelant pour des êtres futurs que l'on veut évoquer au présent par une description de leurs pratiques urbaines.

Si La Città Nuova représente un tournant dans le parcours de l'architecte de Côme en ce qu'elle marque le passage du catalogue de formes solitaires des Dynamismes Architectoniques à une appréhension de la ville comme tissu urbain et collectif, cette métropole du futur est toutefois dépourvue de tout plan d'ensemble: son unité spatiale est dès lors une promesse incarnée par les infrastructures de transport au sein d'un recueil de fragments qui traduisent en clef moderne les principes 


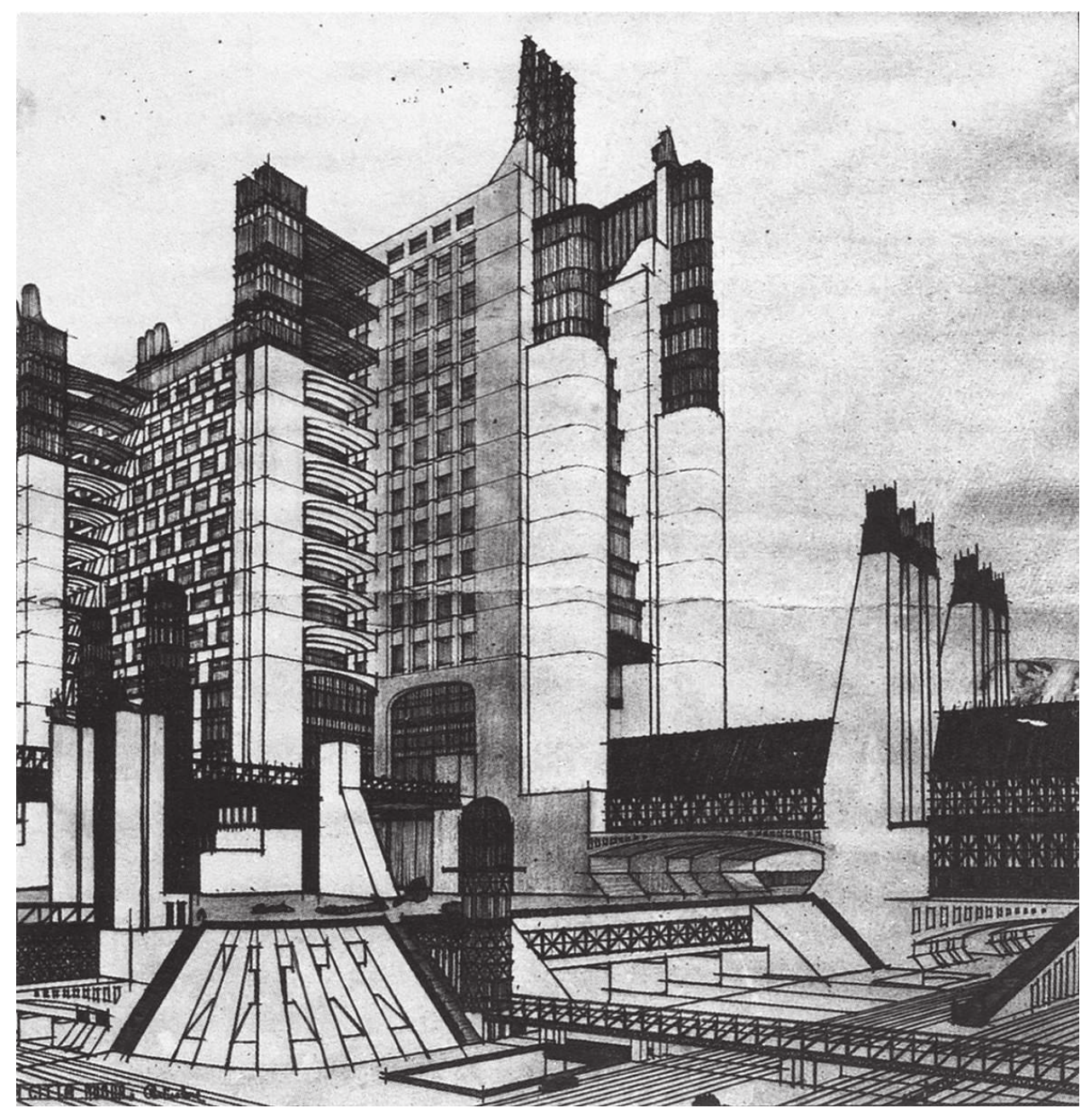

Ill. 9 : Antonio Sant'Elia, « La Città Nuova : casamento con ascensori esterni, galleria, passaggio coperto, su tre piani stradali (linea tramviaria, strada per automobili, passerella metallica), fari e telegrafia senza fili ", 1914, crayon noir-bleu et encre noire, sur papier jaune, 52,5 × 51,5 cm, Museo civico, Côme.

visuels de la veduta, voire du capriccio (ill. 9). C’est précisément dans les vides entre ces dessins s'efforçant de traduire dans un seul geste l'image et son sens ${ }^{20}$, que les projets de La Città Nuova prennent toute leur valeur discursive d'utopie en tant que "résolution historique d'une contradiction [et] synthèse dialectique des contraires ${ }^{21}$. Tendue entre permanences traditionnelles et élans futuristes, détails d'ingénieur et finesses d'artiste, cette ville qui clôture le parcours de l'architecte de Côme à la découverte de la modernité est ainsi une utopie au sens où la concevait Louis Marin : "double figure " de la crise, "représentation ambiguë " de l'écart

20. "L'ingénieur, le cartographe, le peintre, l'architecte, le dramaturge, le poète, en dessinant, peignant, construisant ou parlant le monde, lui offrent son image et son sens, extraient de lui sa véritable réalité dans la représentation qu'ils en donnent "; Marin Louis, Utopiques : jeux d'espaces, Paris, Minuit, 1973, p. 267.

21. Ibid., p. 9. 
productif entre le vrai et le faux, "tableau équivoque de la synthèse possible et de la différenciation productrice; de la réconciliation a venir et de la contradiction agissante : du concept et de l'histoire ${ }^{22}$.

Viviana BIROLLI

doctorante Paris 1/EHESS

22. Ibid., p. 23. 